Reprod. Nutr. Dévelop., 1988, 28 (6 B), 1699-1706

\title{
Hormone de croissance placentaire. Signification par rapport aux hormones de croissance et lactogène
}

G. HENNEN, F. FRANKENNE, Marie-Louise SCIPPO, A. IGOUT, J. CLOSSET, G. PIRENS, Françoise GOMEZ

Endocrinologie expérimentale et clinique, Institut de Pathologie, C.H.U. - B23 - 4000 Liège, Belgique.

Summary. Placental growth hormone. Significance relative to pituitary growth hormones and placental lactogen hormone.

Normal human placenta secretes within maternal compartment a pregnancy associated protein, placental growth hormone $(\mathrm{PGH})$. This entity, agonist of pituitary $\mathrm{GH}$, appears responsible for the elevated IGF I blood levels observed in the mother during pregnancy, while pituitary $\mathrm{GH}$ is no longer secreted. $\mathrm{PGH}$ could thus play a significant role in the anabolic processes of pregnancy. The biochemical mechanism responsible for $\mathrm{PGH}$ production is the expression of the $\mathrm{GH}-\mathrm{V}$ gene at the placental level. This has been demonstrated by the positive probing of $\mathrm{GH}-\mathrm{V}$ mRNA in this tissue, by the establishment of the restriction map and sequence of its cDNA as well as by the NH2-terminal sequence determination of both 22 and 25K PGH forms.

The positive aspect of this function for human reproduction gains weight in that a placenta deleted for the $\mathrm{CS}-\mathrm{A}, \mathrm{GH}-\mathrm{V}$ and $\mathrm{CS}-\mathrm{B}$ gene expresses alternative genes coding for protein similar or related to pituitary $\mathrm{GH}$ or $\mathrm{hPL}$, respectively.

\section{Introduction.}

L'utilisation des technologies nouvelles dans la recherche sur les hormones et facteurs de croissance fait apparaître un domaine de complexité croissante.

Ainsi, le développement d'anticorps monoclonaux anti-somatotropine humaine ( $h \mathrm{GH}$ ) a permis la mise en évidence de la $\mathrm{GH}$ placentaire ( $\mathrm{PGH}$ ) chez la femme enceinte (Hennen et al., $1985 \mathrm{a}, \mathrm{b}, \mathrm{c}$ ). Les progrès dans la connaissance du locus génomique GH-CS (chorio-somatomammotropine ou hPL) (Seeburg, 1982) nous ont amenés à utiliser des techniques de biologie moléculaire (Frankenne et al., 1987 ; Igout et al., 1988) pour l'identification du mécanisme de biosynthèse de $\mathrm{PGH}$. Enfin, pendant que cette entité hormonale prenait place en physiopathologie grâce à l'utilisation de nouvelles méthodes immunologiques de biologie clinique, l'isolement du $\mathrm{PGH}$ à partir du tissu placentaire en révélait deux formes distinctes (Frankenne et al., 1988). Leur séquence NH2-terminale a pu être établie. Les résultats sont présentés ici, établissant de façon ferme la relation entre la protéine $\mathrm{PGH}$ et le gène $\mathrm{GH}-\mathrm{V}$. 
Ce travail passera en revue les différentes étapes d'une approche pluridisciplinaire ayant permis la découverte, l'identification et la signification physiopathologique de l'hormone de croissance placentaire.

\section{L'Hormone de croissance placentaire.}

\subsection{Mise en évidence et physiologie.}

La physiologie de $\mathrm{GH}$ était peu et mal connue pendant la grossesse alors que d'importants processus anaboliques intéressent la mère, l'œuf et l'enfant. C'est au travers de ce souci d'étudier le mode de sécrétion de GH pendant la grossesse que nous avons développé à des fins de dosages immunologiques, des anticorps monoclonaux de haute spécificité et de reconnaissance épitopique connue (Hennen et al., 1985c). Ne présentant aucune réaction avec hPL, ces anticorps ont permis le développement de dosages immunologiques sensibles de $\mathrm{GH}$ dans le sérum de femmes enceintes où, en se rapprochant du terme, hPL et PRL circulent en quantités croissantes. C'est ainsi que nous avons pu démontrer que la sécrétion d'une $\mathrm{GH}$ placentaire $(\mathrm{PGH})$ devenait significative à partir de la $20^{\mathrm{e}}$ semaine, pour augmenter jusqu'au terme, qu'elle était non épisodique et que PGH disparaissait en moins d'une heure du sérum maternel après la délivrance. Pendant que s'affirme la sécrétion de PGH, l'hypophyse maternelle diminue progressivement la sécrétion de $\mathrm{GH}$ qui devient nulle pendant le dernier trimestre de la grossesse.

La place de PGH dans les processus anaboliques de la grossesse a été abordée par l'étude des corrélations entre les taux d'IGF I et de PGH ou de hPL. Le traitement des données fait apparaître une corrélation hautement significative entre PGH et IGF I et non significative entre hPL et IGF I (Caufriez et al., 1988). L'augmentation de taux sérique d'IGFI observée chez la femme enceinte et comparé à celui d'une femme non enceinte peut donc être attribuée à $\mathrm{PGH}$ puisque $\mathrm{GH}$ hypophysaire est mis hors cause.

Le repérage de $\mathrm{PGH}$ dans des fractions de gel filtration de sérums de femmes enceintes montre que cette hormone circule sous forme libre ou liée à des entités qui confèrent au complexe une taille nettement supérieure à celle de PGH. Ceci est à mettre en rapport avec la mise en évidence récente des protéines sériques liant GH hypophysaire et qui, bien que circulantes, sont proches des structures du récepteur à $\mathrm{GH}$ des membranes plasmiques.

PGH est essentiellement secrétée vers le compartiment maternel, le liquide amniotique ne contenant qu'une $\mathrm{GH}$ de type hypophysaire et d'origine foetale (Frankenne et al., 1988).

\subsection{Purification de $P G H$ - Biochimie.}

L'évidence que PGH est une protéine dépendante de la grossesse nous a fait étudier le placenta comme source naturelle de l'hormone. Le schéma d'extraction, de purification et de chromatographie sur DEAE-cellulose s'est inspiré de celui 
utilisé pour GH hypophysaire et hPL (Frankenne et al., 1988). Une fraction enrichie en PGH est ainsi obtenue. Le comportement chromatographique de PGH se montre plus basique que celui de GH hypophysaire et hPL. Cette fraction a été ensuite traitée par chromatographie d'affinité sur un support auquel est couplé l'anticorps monoclonal la reconnaissant spécifiquement. L'éluat a été analysé par électrophorèse en gel de polyacrylamide - SDS. Après électro-transfert, les protéines ont été révélées sur filtre de nitrocellulose par une réaction immunoenzymatique spécifique (fig. 1 ). Deux formes de poids moléculaires 22 et $25 \mathrm{~K}$ sont mises en évidence.

\section{SDSPAGE (15\%) DE PGH "WESTERN-BLOT"-5B4 MAb}

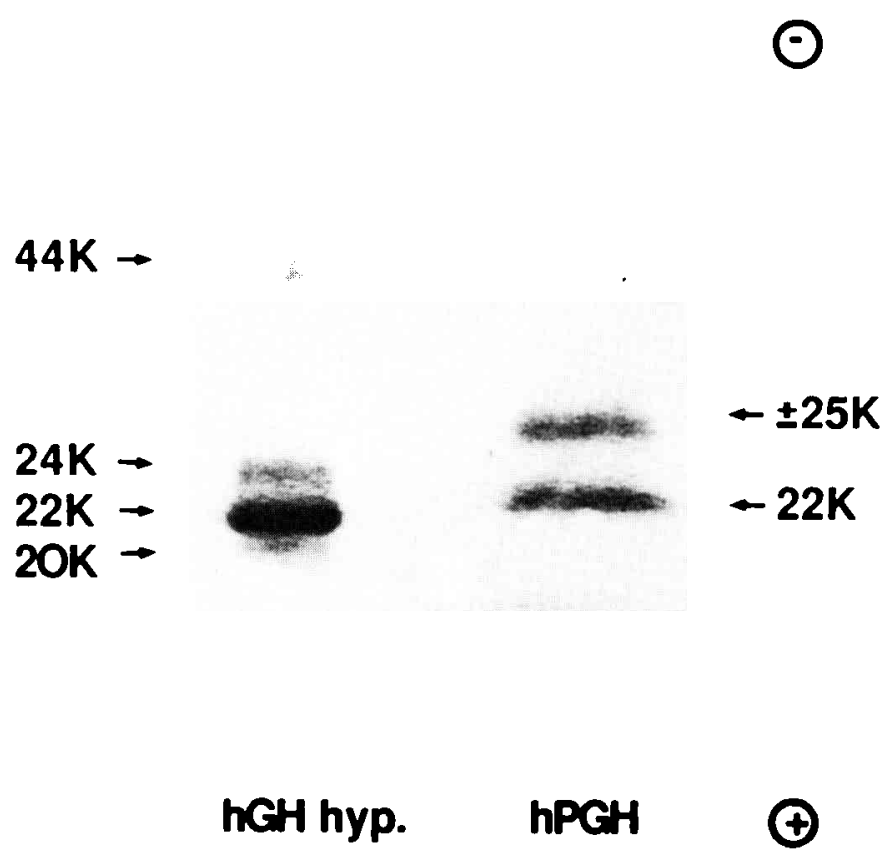

FIG. 1. - "Western blot" du matériel adsorbé sur la colonne d'affinité.

Dans une autre expérience, ces formes ont été transférées sur filtre PVDF (Millipore) et soumises individuellement à l'analyse de séquence $\mathrm{NH} 2$-terminale par un procédé de microdétermination sur 15 picomoles approximativement (Applied Biosystem model 477 A sequencer). La figure 2 donne la séquence des 29 acides aminés NH2-terminaux obtenue pour ces deux formes. Elles apparaissent identiques et montrent par rapport à la séquence $\mathrm{NH} 2$-terminale de $\mathrm{GH}$ hypophysaire, trois mutations caractéristiques.

Reproduction, Nutrition, Développement, $n^{\circ} 6 \mathrm{~B} / 88-12$ 
P-GH (22-25K) GH-N (hypoph.)
Phe-Pro-Thr-lle-Pro-Leu-Ser-Arg-Leu-PhePhe-Pro-Thr-lle-Pro-Leu-Ser-Arg-Leu-Phe-

15 20 Asp-Asn-Ala-Met-Leu-Arg-Ala Arg-Arg-Leu-
Asp-Asn-Ala-Met-Leu-Arg-Ala His-Arg-Leu25 Tyr-GIn-Leu-Ala Tyr-Asp-Thr-Tyr-GIn-
His-GIn-Leu-Ala -Phe-Asp-Thr-Tyr-GIn-

FIG. 2. - Séquences NH2-terminales des formes 22 et $25 \mathrm{~K}$ de PGH comparées à celle de GH-N hypophysaire.

Une glycosylation a été recherchée par chromatographie d'affinité des deux formes sur Concanavaline A-Sepharose. La forme $22 \mathrm{~K} \mathrm{PGH}$ est partiellement non adsorbée. Une fraction significative des deux formes 22 et $25 \mathrm{~K}$ est adsorbée et fait retenir l'hypothèse d'une glycosylation d'une proportion des deux formes.

Enfin, l'étude de PGH a montré que cette hormone interagissait comme compétiteur puissant de $\mathrm{GH}$ hypophysaire avec les récepteurs à $\mathrm{GH}$ du foie de la lapine gravide (Frankenne et al., 1988).

2.3 Expression des gènes de la famille GH-CS et identification de leur produit.

Le locus $\mathrm{GH}-\mathrm{CS}$ comprend 5 gènes dont le gène $\mathrm{GH}-\mathrm{N}$ exprimé au niveau hypophysaire et dont le produit est la GH connue. Les positions $5^{\prime}-3^{\prime}$ s'ordonnent comme indiqué dans la figure 3 . Le gène $h C S-L$ est présumé non fonctionnel. Notre attention s'est immédiatement portée sur le gène $\mathrm{GH}-\mathrm{V}$ présumé non exprimé jusqu'il y a peu. Sa structure implique un variant de $\mathrm{GH}$, plus basique que $\mathrm{GH}$ hypophysaire, et présentant 13 substitutions d'acides aminés. Ce gène, transfecté en cellule d'eucaryotes, avait exprimé une protéine dont les caractéristiques se rapprochaient de celles décrites ici pour PGH (Pavlakis et al., 1981). Nous avons dès lors utilisé la technique d'hybridation pour mettre en évidence la présence du mRNA du gène $V$ au niveau placentaire (Frankenne et al., 1987) afin d'en démontrer l'expression dans ce tissu.

Les sondes oligonucléotidiques ( $V, N$ ou $C S$ ) utilisées ont été sélectionnées pour leur spécificité de façon à s'assurer par comparaison de signaux, l'identification du mRNA spécifique du gène $V$ (tabl. 1). Par "Northern Blotting ", nous mettons en évidence un mRNA d'environ $1 \mathrm{~Kb}$ spécifiquement reconnu par la 


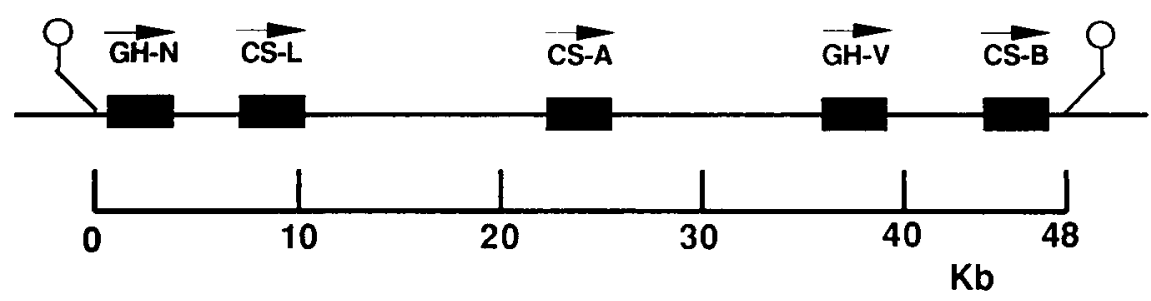

$Q=E c o \mathbf{R} \mathbf{l}$

FIG. 3. - Locus des gènes hGH-hCS. (Seeburg, 1987).

\section{'NORTHERN BLOT"}

\section{SONDE N}

\section{SONDE V}

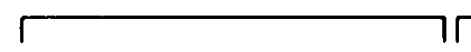

\section{$0.85 \mathrm{~Kb}-$}
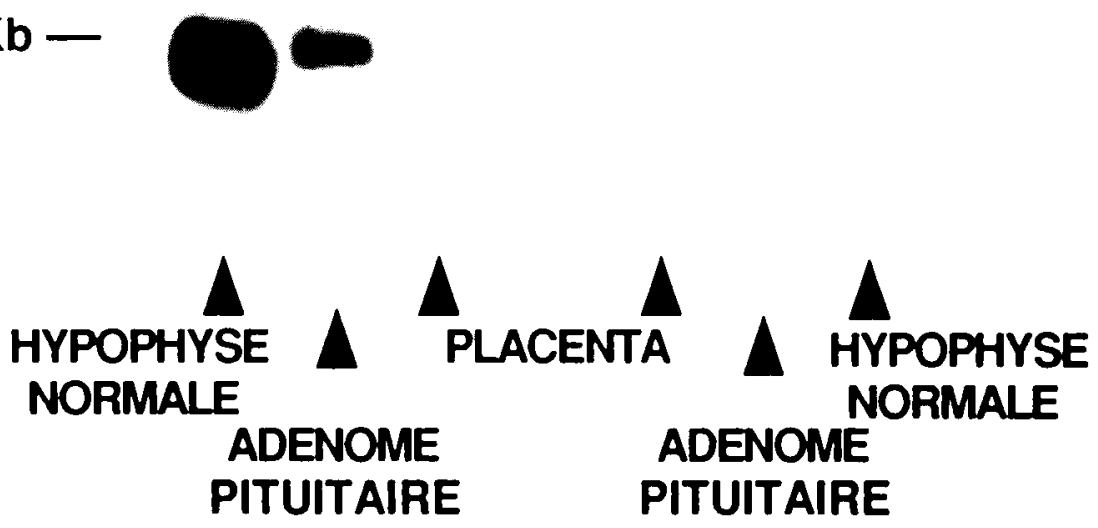

FIG. 4. - Hybridation de mRNA placentaire et hypophysaire par les sondes $N$ et $V$.

sonde $V$ et uniquement dans l'extrait placentaire. D'autre part, des mRNA d'hypophyses normales ou ceux d'un adénome à $\mathrm{GH}$ ne sont reconnus que par la sonde $\mathrm{N}$.

Nous avons ensuite utilisé une banque de cDNA placentaires humains insérés dans le site de restriction EcoRI du $\lambda$ gt 11 (Clontech, Palo Alto, USA) pour clôner le cDNA du PGH et en déterminer la séquence (lgout et al., 1988). 
TABLEAU 1. - Sondes oligonucléotidiques de 15 bases.

\begin{tabular}{|c|c|c|c|c|}
\hline \multirow[t]{2}{*}{ OLIGO } & \multirow{2}{*}{$\begin{array}{l}\text { SEQUENCE CORRESPONDANTE } \\
\text { EN ACIDES AMINES }\end{array}$} & \multicolumn{3}{|c|}{ NON CORRESPONDANCE AVEC LES GENES } \\
\hline & & CS-A-B & GH-N & GH-V \\
\hline & 110 & & & \\
\hline V & & $\star \star \star \star \star$ & $\star \star \star \star \star$ & \\
\hline & 114 & & & \\
\hline $\mathbf{N}$ & & * & & $\star \star \star \star$ \\
\hline & -3 & & & \\
\hline 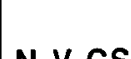 & 126 & & & \\
\hline$n-V-C S$ & & & & \\
\hline
\end{tabular}

Les phages recombinants ont été repérés par l'utilisation des sondes du tableau 1. Après hybridation, trois clones se sont révélés positifs avec les trois sondes. La digestion du DNA de ces recombinants par EcoRI a produit un fragment de $800 \mathrm{pb}$, qui était la taille présumée du cDNA de hGH-V. La carte de restriction de ce cDNA est en accord avec celle du gène $\mathrm{GH}-\mathrm{V}$ si l'on retient le mode d'épissage proposé par Seeburg (1982). La séquence des cDNA de GH-V a ensuite été établie et confirme l'épissage du gène hGH-V (Igout et al., 1988).

La structure primaire du produit de ce gène correspond bien à la séquence NH2-terminale de PGH établie par nous et révèle en outre que le site de glycosylation en position 140-141-142 (Asn-Gln-Ser) décrit antérieurement pour le produit du gène GH-V (Seeburg, 1982) est bien présent.

L'ensemble de ces résultats établit l'identité de PGH avec GH-V. Cooke $e t$ al. (1988) ont repéré un mode d'épissage alternatif du gène aboutissant à une forme de cDNA contenant un segment de 253 nucléotides correspondant au $4^{e}$ intron du gène $V$. Un éventuel produit d'expression correspondant à cette structure de mRNA aboutirait à une protéine variante contenant une séquence $\mathrm{NH} 2$-terminale de 65 acides aminés commune à la PGH décrite par nous et se poursuivant par 104 résidus codés par le $4^{\mathrm{e}}$ intron du gène. Le poids moléculaire de ce variant serait ainsi bien supérieur à $22 \mathrm{~K}$ et le site de glycosylation serait perdu. Comme par ailleurs notre isolement des formes 22 et $25 \mathrm{~K}$ de $\mathrm{PGH}$ repose sur I'utilisation d'anticorps monoclonaux reconnaissant des épitopes précis, il n'est pas exclu que notre méthode d'extraction n'ait pas retenu le produit hypothétique correspondant à ce mode d'épissage et dont les caractéristiques physicochimiques doivent être très différentes de celles de la PGH décrite par nous. 


\section{Délétion des gènes $\mathrm{CS}-\mathrm{A}, \mathrm{GH}-\mathrm{V}$ et $\mathrm{CS}-\mathrm{B}$.}

A travers l'observation de grossesses évoluant normalement mais avec un taux nul ou très bas d'hPL sérique chez la mère, des délétions du locus CS-GH ont été identifiés par Parks et al. (1985), Wurzel et al. (1982) et Simon et al. (1986).

Nous avons étudié les protéines de la famille GH-CS qui étaient exprimées dans un placenta porteur de la délétion des gènes $\mathrm{CS}-\mathrm{A}, \mathrm{GH}-\mathrm{V}$ et $\mathrm{CS}-\mathrm{B}$ (Wurzel et al., 1982) et où on devait s'attendre à un défaut de PGH et hPL. Combinant les méthodes d'extraction et de purification utilisées pour $\mathrm{PGH}$ et les dosages immunologiques spécifiques permettant d'identifier hPL, GH-N et PGH, nous avons démontré la présence de facteurs de type GH-N et CS dans ce type de placenta. Cependant, le facteur présentant une immunoréactivité de type hPL (CS) se comporte en chromatographie différemment de hPL normale.

Les deux types de facteurs (GH-N et $\mathrm{CS}$ ) se sont révélés capables de se lier au récepteur hépatique de $\mathrm{GH}$ dont ils sont des agonistes complets. L'hypothèse serait que des gènes silencieux ou absents dans le génome normal et au niveau placentaire tel le gène $\mathrm{GH}-\mathrm{N}$ et un gène de type $\mathrm{CS}$ s'exprimerait dans les placenta porteurs de la délétion CS-A-GH-V et CS-B (Hennen et Frankenne, 1987).

\section{Conclusions.}

Nous avons démontré que le placenta humain normal secrète dans le compartiment maternel une hormone spécifique de la grossesse, la GH placentaire $(\mathrm{PGH})$. Celle-ci, agoniste de $\mathrm{GH}$ hypophysaire, semble bien activer l'IGF I maternelle pendant que cesse la sécrétion hypophysaire de la $\mathrm{GH}$. Ceci laisse présumer le rôle de PGH sur l'anabolisme de la grossesse. Le mécanisme responsable de cette sécrétion est l'expression au niveau placentaire du gène $\mathrm{GH}-\mathrm{V}$. Ceci a été démontré par la révélation dans ce tissu, du mRNA correspondant, par l'établissement de la carte de restriction et de la séquence du cDNA et enfin par l'établissement de la séquence $\mathrm{NH} 2$-terminale des deux formes moléculaires 22 et $25 \mathrm{~K}$ de PGH.

L'étude d'un placenta porteur d'une délétion touchant les gènes CS-A-GH-V et CS-B a montré que s'exprimaient des protéines alternatives parentes ou identiques à la GH hypophysaire ainsi que d'autres proches de hPL.

$27^{e}$ Réunion de la Société française pour l'Etude de la Fertilité. Paris, 29, 30 sept., $1^{\text {er }}$ oct. 1988.

Remerciements. - Nous désirons exprimer notre reconnaissance pour la collaboration du Professeur Lambotte et de son cadre (Gynécologie-Obstétrique, Université de Liège). Les séquences $\mathrm{NH} 2$-terminales ont été réalisées par le Dr. J. Van Beumen (Laboratorium voor Microbiologie, Université de Gand).

Le tissu placentaire porteur de la délétion nous a été procuré par les Drs. Nielsen, P. V. (Danemark) et Parks, J.S. (USA). La délétion est décrite par Wurzel et al. (1982).

Ce travail a reçu le soutien financier de la Région Wallonne, du FRSM et de I'IRSIA. 


\section{References}

CAUFRIEZ A., FRANKENNE F., HENNEN G., GOLSTEIN J., CANTRAINE F., ENGLERT $Y$., COPINSHI G., 1987. Possible mechanisms of somatomedin-C elevation during normal human pregnancy. 1st Eur. Congr. Endocrinol., Copenhagen, Denmark (Abstract 21).

COOKE N. E., RAY J., EMERY J. G., LIEBHABER S. A., 1988. Two distinct species of human growth hormone-variant mRNA in the human placenta predict the expression of novel growth hormone proteins. J. biol. Chem., 263, $9001-9006$.

FRANKENNE F., RENTIER-DELRUE F., SCIPPO M. L., MARTIAL J., HENNEN G., 1987. Expression of the growth hormone variant gene in human placenta. J. clin. Endocrinol. Metab., 64, 635-637.

FRANKENNE F., CLOSSET J., GOMEZ F., SCIPPO M.L., SMAL J., HENNEN G., 1988. The physiology of growth hormones $(\mathrm{GHs})$ in pregnant women and partial characterization of the placental GH variant. J. clin. Endocrinol. Metab., 66, 1171-1180.

HENNEN G., FRANKENNE F., PIRENS G., GOMEZ F., CLOSSET J., SCHAUS Ch., EL KHAYAT N., 1985a. New chorionic $\mathrm{GH}$-like antigen revealed by monoclonal antibody radioimmunoassays. Lancet, 1, 399.

HENNEN G., FRANKENNE F., PIRENS G., GOMEZ F., EL KHAYAT N., CLOSSET J., SCHAUS Ch., 1985b. A chorionic $\mathrm{GH}$-like antigen : increasing levels during second half of pregnancy with pituitary $\mathrm{GH}$ suppression as revealed by monoclonal antibody radioimmunoassays. Int. J. Fertil., 30, 27-33.

HENNEN G., FRANKENNE F., CLOSSET J., GOMEZ F., PIRENS G., SMAL J., BECKERS A., EL KHAYAT N., LAMBOTTE R., 1985c. Monoclonal antibody to growth hormone: the discovery of a new variant, human placental growth hormone. Vol. 30, 29-40. In FORTI G., SERIO M., LIPSETT M. B., Florence, Italy. Int. Symp. on Monoclonal antibodies : basic principles, experimental and clinical applications in endocrinology. ARES Serono Symp., Raven Press, New York.

HENNEN G., FRANKENNE F., 1987. Influence des hormones protéiques placentaires sur la physiologie maternelle. Ann. Endocrin. (Paris), 48, 278-288.

IGOUT A., SCIPPO M. L., FRANKENNE F., HENNEN G., 1988. Cloning and nucleotide sequence of placental hGH-V cDNA. Arch. int. Physiol. Biochim., 96, 63-67.

PARKS J. S., NIELSEN P. V., SEXTON L. A., JORGENSEN E. H., 1985. An effect of gene dosage on production of human chorionic somatomammotropin. J. clin. Endocrinol. Metab., 60, 994-997.

PAVLAKIS G. N., HIZUKA N., GORDEN P., SEEBURG P., HAMER D. H., 1981. Expression of two human growth hormone genes in monkey cells infected by simian virus 40 recombinants. Proc. nat. Acad. Sci. USA, 78, 7398-7402.

SEEBURG P. H., 1982. The human growth hormone gene family: nucleotide sequences show recent divergence and predict a new polypeptide hormone. DNA, 1, 239-249.

SIMON Ph., DECOSTER C., BROCAS H., SCHWERS J., VASSART G., 1986. Absence of human chorionic somatomammotropin during pregnancy associated with two types of gene deletion. Hum. Genet, 74, 235-238.

WURZEL J. M., PARKS J., HERD J. E., NIELSEN P. V., 1982. A gene deletion is responsible for absence of human chorionic somatomammotropin. DNA, 1, 251-257. 